\title{
Chip-scale optical airflow sensor
}

\author{
Yumeng Luo ${ }^{1}$, Xiaoshuai $\mathrm{An}^{1}$, Liang Chen ${ }^{1}$ and Kwai Hei Li ${ }^{1,2,3 凶}$
}

\begin{abstract}
Airflow sensors are an essential component in a wide range of industrial, biomedical, and environmental applications. The development of compact devices with a fast response and wide measurement range capable of in situ airflow monitoring is highly desirable. Herein, we report a miniaturized optical airflow sensor based on a GaN chip with a flexible PDMS membrane. The compact GaN chip is responsible for light emission and photodetection. The PDMS membrane fabricated using a droplet-based molding process can effectively transform the airflow stimuli into optical reflectance changes that can be monitored by an on-chip photodetector. Without the use of external components for light coupling, the proposed sensor adopting the novel integration scheme is capable of detecting airflow rates of up to $53.5 \mathrm{~ms}^{-1}$ and exhibits a fast response time of $12 \mathrm{~ms}$, holding great promise for diverse practical applications. The potential use in monitoring human breathing is also demonstrated.
\end{abstract}

\section{Introduction}

The velocity measurement and distribution analysis of airflow is of great significance in the fields of atmospheric environmental monitoring, aerodynamic studies, turbine inspection, navigation control, biomedical engineering, and so on ${ }^{1-5}$. Sensing devices employing the principles of thermoresistance ${ }^{6-9}, \quad$ piezoresistance ${ }^{10-13}$, electrical resistance $^{14-16}$, capacitance ${ }^{17}$, magnetoelasticity ${ }^{18}$, and mechanoluminescence ${ }^{19}$ have been developed for airflow detection. However, the achievement of a sensor with both fast response and a wide measurement range remains an unsolved challenge. Recently, optical airflow sensors based on fiber optics have attracted substantial research interest due to their distinctive advantages, such as their lightweight, high sensitivity, and fast optical response $^{20,21}$. To improve the sensitivity of sensors to optical changes induced by airflow, several advanced architectures, such as fiber Bragg gratings $^{22,23}$, Fabry-Pérot interferometers ${ }^{24,25}$, microcantilevers ${ }^{26}$, and other nanostructures ${ }^{27}$, have been incorporated. Nevertheless, the reported fiber-optic sensing systems mostly

\footnotetext{
Correspondence: Kwai Hei Li (khli@sustech.edu.cn)

${ }^{1}$ School of Microelectronics, Southern University of Science and Technology, Shenzhen 518055, China

${ }^{2}$ Engineering Research Center of Integrated Circuits for Next-Generation

Communications, Ministry of Education, Southern University of Science and

Technology, Shenzhen 518055, China

Full list of author information is available at the end of the article
}

involve the complex assembly of external components and require precise optical alignment to couple the light from a light source to a spectroscopic analyzer through optical fiber as the sensing medium. The bulky and complex configurations inevitably weaken the efficiency, robustness, and compactness of the system and limit its scope of applications. Furthermore, despite their intrinsic fast response based on optical means, the response time of the reported optical systems in response to airflow has not been investigated and presented.

Eliminating the external light-coupling components is a promising approach for realizing the miniaturization of optical airflow sensors. Considering the materials used to construct solid-state high-performance optical devices, GaN-based direct bandgap semiconductors and their alloys are excellent candidates because of their high efficiency, fast transient response, good physical and chemical stability, and long operational lifespan ${ }^{28,29}$. Recently, the monolithic integration of GaN-based optical and electronic components on a chip-scale platform has been demonstrated for on-chip visible light communication ${ }^{30,31}$, heart rate detection ${ }^{32}$, and illumination and imaging ${ }^{33}$. However, to date, reports on the use of integrated GaNbased devices for airflow detection remain extremely limited. The major limitation is that the existence of rigid and hard growth substrates, such as sapphire and silicon, limits the deformation of the device in response to airflow. 
The only report about a GaN-based airflow sensor employs a suspended $\mathrm{GaN}$ membrane that shows a detectable airflow rate of up to $2.779 \mathrm{~ms}^{-1}$ and a switch-on time of $1 \mathrm{~s}^{34}$. Although a bendable GaN film can be realized by the selective wet etching of silicon or laser lift-off of sapphire ${ }^{35,36}$, the use of a free-standing GaN film as a sensing medium responding to high airflow is less reliable due to its fragility. Moreover, structural deformation introduces unwanted strain in the $\mathrm{GaN}$ epitaxial layer, which degrades the internal quantum efficiency of the device $^{36}$. Therefore, a highly flexible structure should be explored as an alternative sensing medium.

In this work, a novel compact integration of a GaN chip with a flexible PDMS membrane that enables the chip to sense airflow without the need for external light coupling components is proposed, as shown in Fig. 1a. The GaN chip with the dual functions of emission and detection is fabricated on a $\mathrm{GaN} /$ sapphire template through waferscale fabrication processes, while the PDMS membrane is formed by a low-cost droplet-based molding process. The electrical and optical properties of the on-chip device, as well as its ability to detect a wide range of airflow rates, are studied to confirm the effectiveness of the proposed integration scheme.

\section{Results and discussion}

GaN chips are fabricated through wafer-scale microfabrication processes, including photolithography, etching, and deposition of metal and oxide layers. The structural layout of the GaN chip is schematically shown in Fig. 1b. The PDMS membrane structure is fabricated by a molding process using water droplets as a template, as depicted in Fig. 1c. With the highly sticky nature of the PDMS surface, the Al film can be adhered firmly to the PDMS membrane, as shown in Fig. 1d, e. The PDMS block is then fixed on the GaN chip by gluing the film edges with the PDMS gel, followed by a curing process. The packaged chip integrated with the PDMS membrane is shown in Fig. 1f-h. The electrical connection to the on-chip devices can be established through the $\mathrm{Al}$ printed circuit board (PCB) package; that is, the LED can be biased by a current source, and the photocurrent current can be read out by an ammeter.

The working principle of the proposed airflow sensor is shown in Fig. 1a. Under current injection, the LED emits light as the carriers confined in the InGaN active region recombine radiatively. The bottom distributed Bragg reflector (DBR) prompts the emitted light to radiate upward. The light extracted from the transparent sapphire propagates toward the $\mathrm{Al}$ film. When airflow is applied, the PDMS membrane together with the $\mathrm{Al}$ film is deformed and modulates the amount of reflected light reaching the PD. The light is absorbed by the InGaN layer in the PD, and the converted photocurrent signals can be used to indicate the airflow change.

Formed on a single chip containing an InGaN/GaN multiquantum well (MQW), the LED and PD are the core elements responsible for light emission and detection,

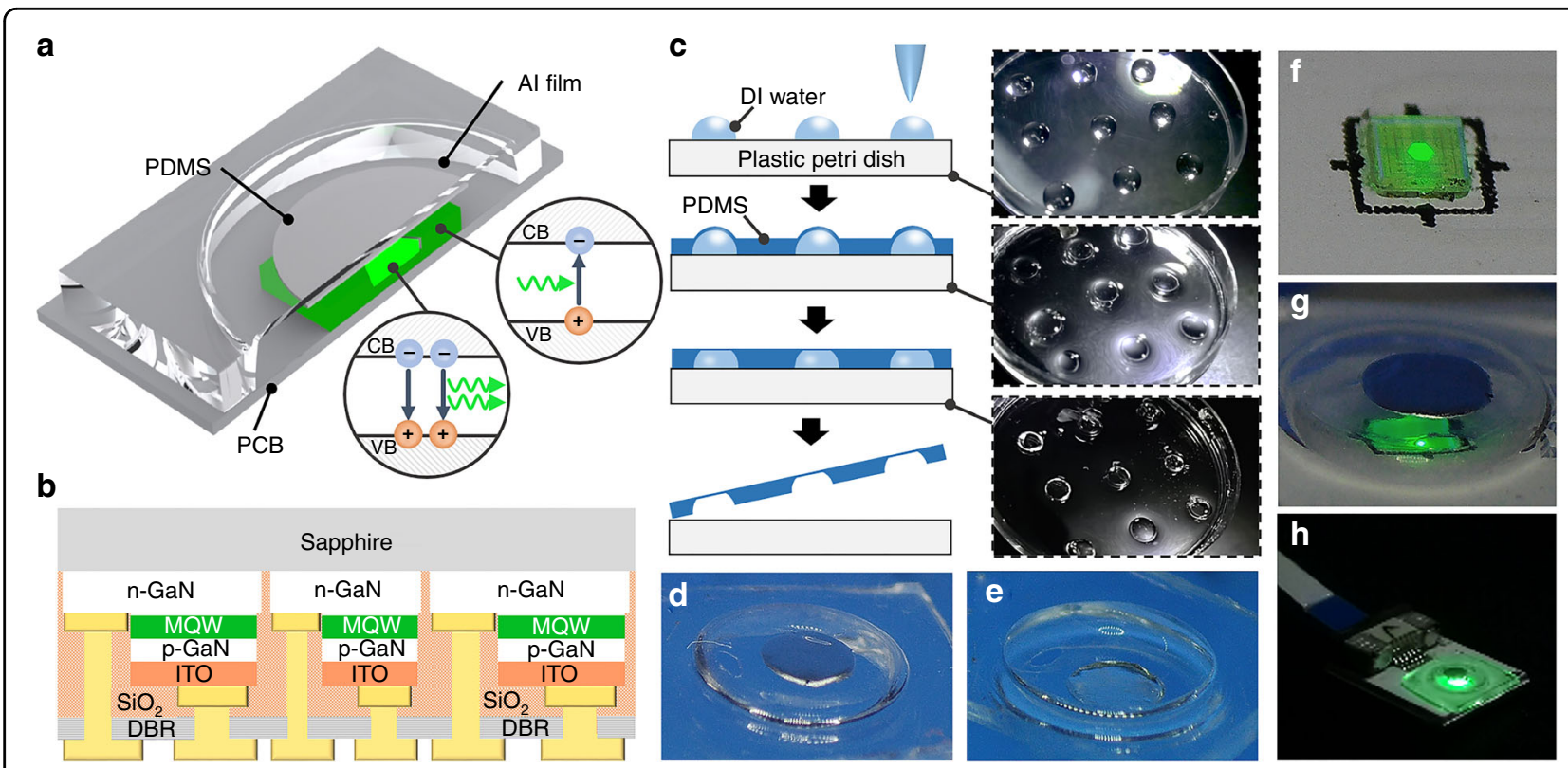

Fig. 1 Structure of the optical airflow sensor. Schematic diagrams of the a proposed airflow sensor and $\mathbf{b}$ structural layout of the GaN chip. c Schematic illustrations of the fabrication process of the PDMS membrane and the corresponding optical images at different stages.

Microphotographs of the $\mathbf{d}$ frontside and $\mathbf{e}$ backside of the PDMS membrane with Al film. Magnified image of the packaged chip $\mathbf{f}$ before and $\mathbf{g}$ after coverage of the PDMS membrane. $\mathbf{h}$ Optical image of the resultant airflow sensor 

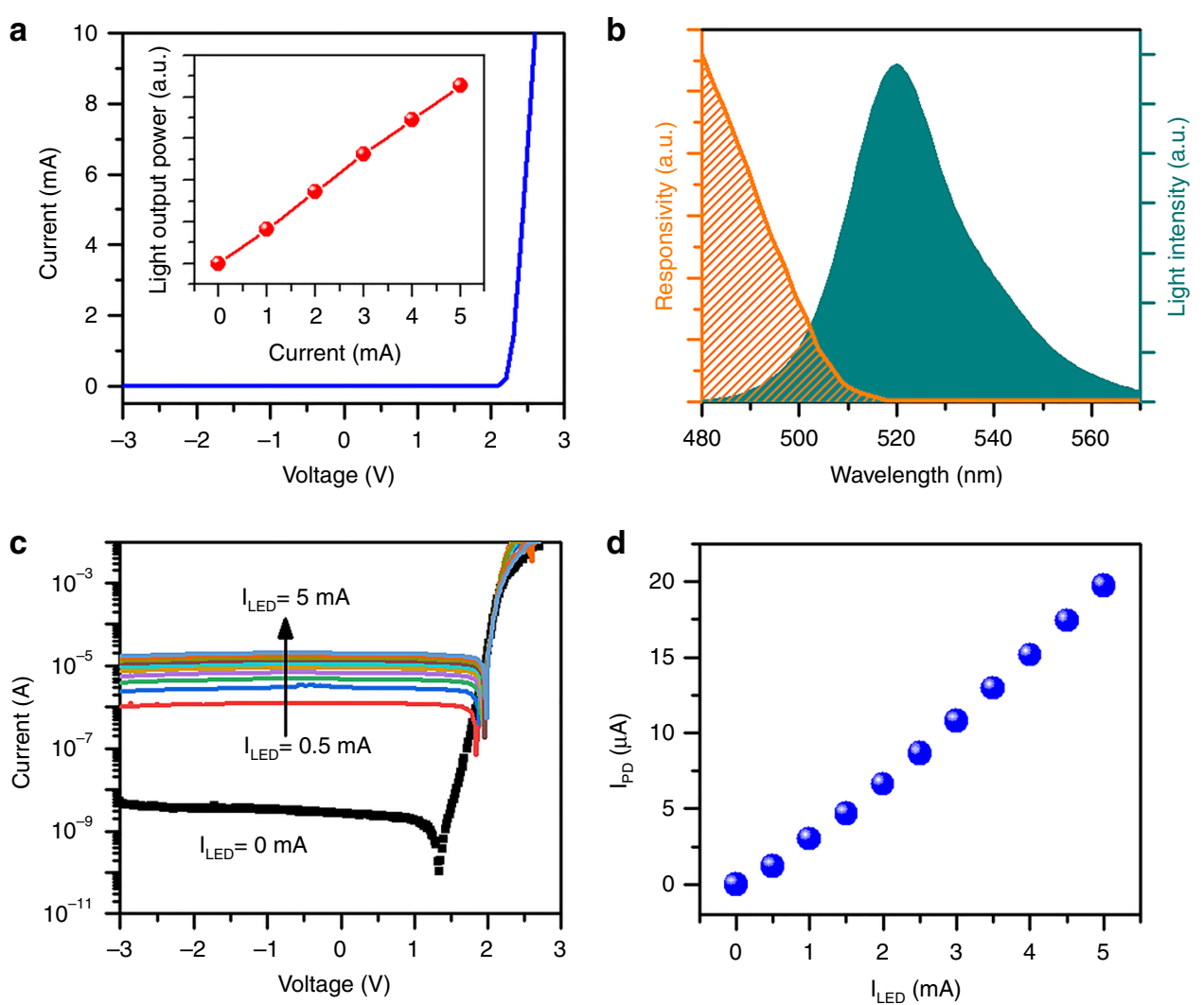

Fig. 2 Optical and electrical properties of the GaN chip. a $I-V$ characteristics of the LED. Inset showing a graph of the light output power as a function of the current. $\mathbf{b}$ EL spectra of the biased LED and spectral responsivity of the unbiased PD. $\mathbf{c} I-V$ curves of the PD measured under different LED currents. $\mathbf{d}$ Plot of the PD photocurrent as a function of the LED current

respectively, and their optical and electrical properties are characterized. Fig. 2a shows the current-voltage $(I-V)$ curve of the LED measured using a source measurement unit (Keithley 2450), which provides a current resolution of $50 \mathrm{pA}$. The forward-biased voltage is $2.44 \mathrm{~V}$ at $5 \mathrm{~mA}$, and the resistance calculated from the inverse slope of the $I-V$ curve is $33.2 \Omega$. The inset of Fig. 2a reveals that the light output power increases with the bias current. With an identical InGaN-based MQW diode structure for photodetection in the PD, the response of PD to the LED emission is then verified. From the electroluminescence (EL) spectra of the LED plotted in Fig. 2b, the peak wavelength and spectral width are 521.5 and $26.8 \mathrm{~nm}$ at an LED current of $5 \mathrm{~mA}$, respectively. It can be seen from the same figure that the responsivity curve of PD decreases as the wavelength increases and overlaps with the emission spectrum of approximately $30 \mathrm{~nm}$. The observation of the partial spectral overlap is attributed to the absorption edge shift related to the quantum-confined stark effect and the band tail effect arising from the indium fluctuation of the MQW ${ }^{37}$. Measured under no-airflow conditions, the $I-V$ curves of the PD under different LED currents are plotted in Fig. 2c. In the absence of LED illumination, the photocurrents measured at reverse bias voltages are at a low level, on the order of $10^{-9} \mathrm{~A}$. When an injection current of $5 \mathrm{~mA}$ is applied to the LED, the PD photocurrent rises significantly, by more than 5 orders of magnitude to $10^{-5} \mathrm{~A}$. As observed from the currentcurrent plot in Fig. 2d, a linear proportional relationship suggests that the photocurrent can highly respond to the intensity change of the LED emission.

The photocurrent responses of the device at different airflow rates are acquired, and the measurement setup is illustrated in Fig. 3a. The inlet of the air pipe is connected to an airflow generator, and its outlet is mounted on a linear motorized stage. By controlling the position of the pipe outlet, the airflow experienced by the sensor can be adjusted. The airflow rate readings are calibrated using a commercial airflow meter (Taicang Huayu, WS200B). During the measurement, the LED is biased at a constant current of $5 \mathrm{~mA}$, and the PD remains zero biased. Fig. 3b plots the measured photocurrents at different airflows of up to $53.5 \mathrm{~ms}^{-1}$. By performing linear regression on the data, we determine that the slopes of the fitted lines for increasing and decreasing airflow are 0.2664 and $0.2686 \mu \mathrm{A} /\left(\mathrm{ms}^{-1}\right)$, respectively, and both $\mathrm{R}$-squared 


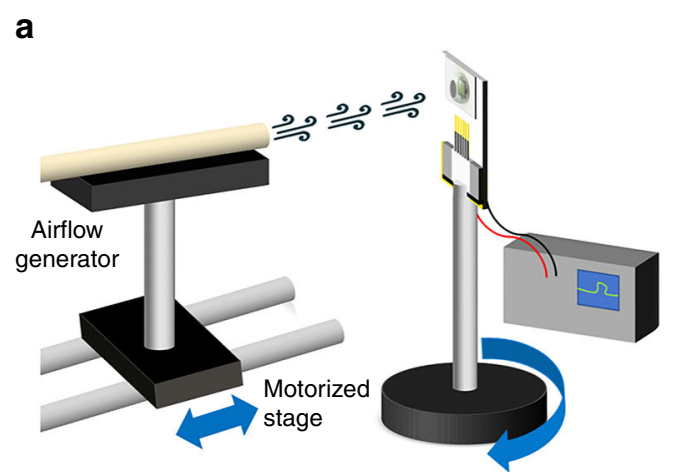

C
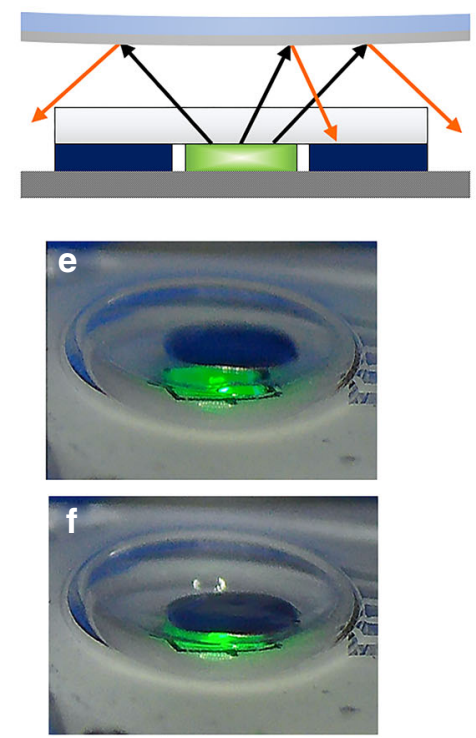

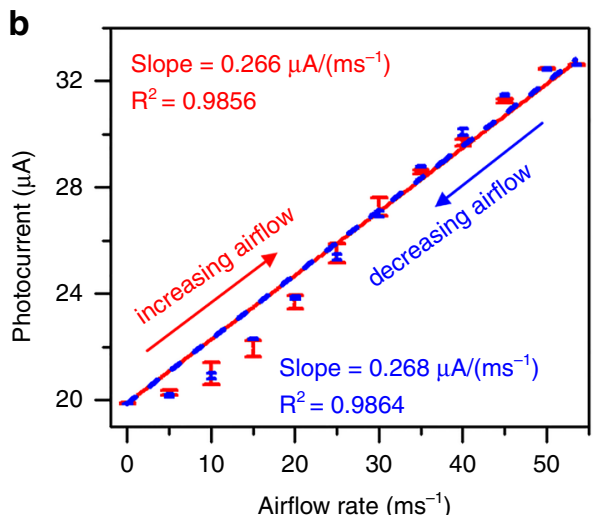

d
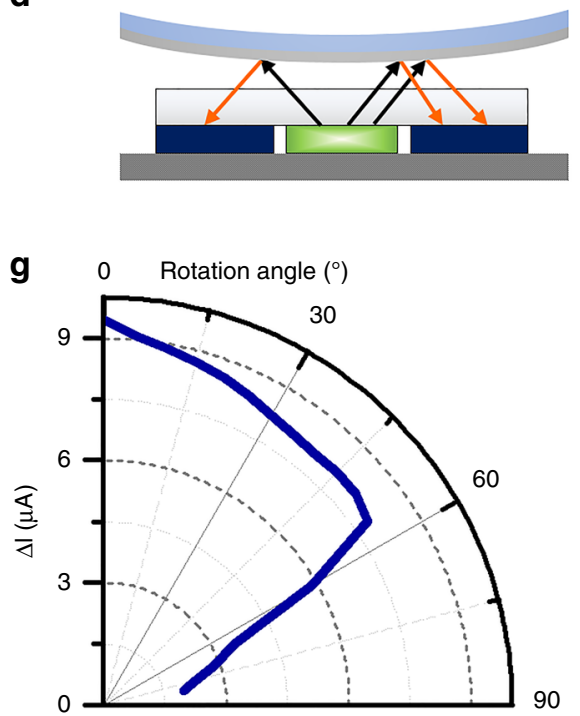

Fig. 3 Response of the optical sensor to airflow. a Schematic diagram showing the setup for measuring the sensor response to airflow. $\mathbf{b}$ Plot of the photocurrent as a function of the airflow rate. Schematic illustrations of the distribution of reflected light under $\mathbf{c}$ low and $\mathbf{d}$ high airflows. Optical images of the sensor were taken under $\mathbf{e}$ low and $\mathbf{f}$ high airflows. $\mathbf{g}$ Polar plot showing the photocurrent response of the sensor at varying rotation angles

values are larger than 0.98. Under low airflow, the PDMS membrane deforms less and is highly separated from the chip, as illustrated in Fig. 3c. With the divergence characteristics of the LED emission, the large spacing reduces the portion of reflected light reaching the PD. When increasing the airflow rate, the PDMS membrane together with the $\mathrm{Al}$ film is pushed toward the chip, resulting in more light being received by the $\mathrm{PD}$, as depicted in Fig. 3d. Under the maximum detectable airflow of $53.5 \mathrm{~ms}^{-1}$, the PDMS membrane together with the Al film is very close to the chip surface. The photocurrent remains unchanged when further increasing the airflow beyond $53.5 \mathrm{~ms}^{-1}$. Furthermore, it can be seen from the captured image shown in Fig. 3e, $\mathrm{f}$ that the PDMS membrane deforms more at higher airflow. It is worth noting that there exists a stable background photocurrent of approximately $20 \mu \mathrm{A}$ that does not respond to the applied airflow. This background signal originates from the light guided inside the chip rather than the externally reflected light. With the refractive index contrast of the sapphire/air interface at the chip surface, the emitted light with an angle of incidence larger than the critical angle of approximately $34.4^{\circ}$ undergoes total internal reflection and is partially coupled into the PD through the transparent sapphire.

To investigate the effects of different airflow directions on the photocurrent response, the sensor is mounted on a motorized rotation stage. The airflow rate is fixed at $38.7 \mathrm{~ms}^{-1}$, and the photocurrents are collected from $0^{\circ}$ to $80^{\circ}$ with a step size of $10^{\circ}$. From the results plotted in Fig. 3g, it is observed that the photocurrent decreases gradually from $9.44 \mu \mathrm{A}$ to $7.88 \mu \mathrm{A}$ when the sensor is rotated from $0^{\circ}$ to $80^{\circ}$. When the angle is further increased to $80^{\circ}$, the photocurrent change drops rapidly to $1.95 \mu \mathrm{A}$, 

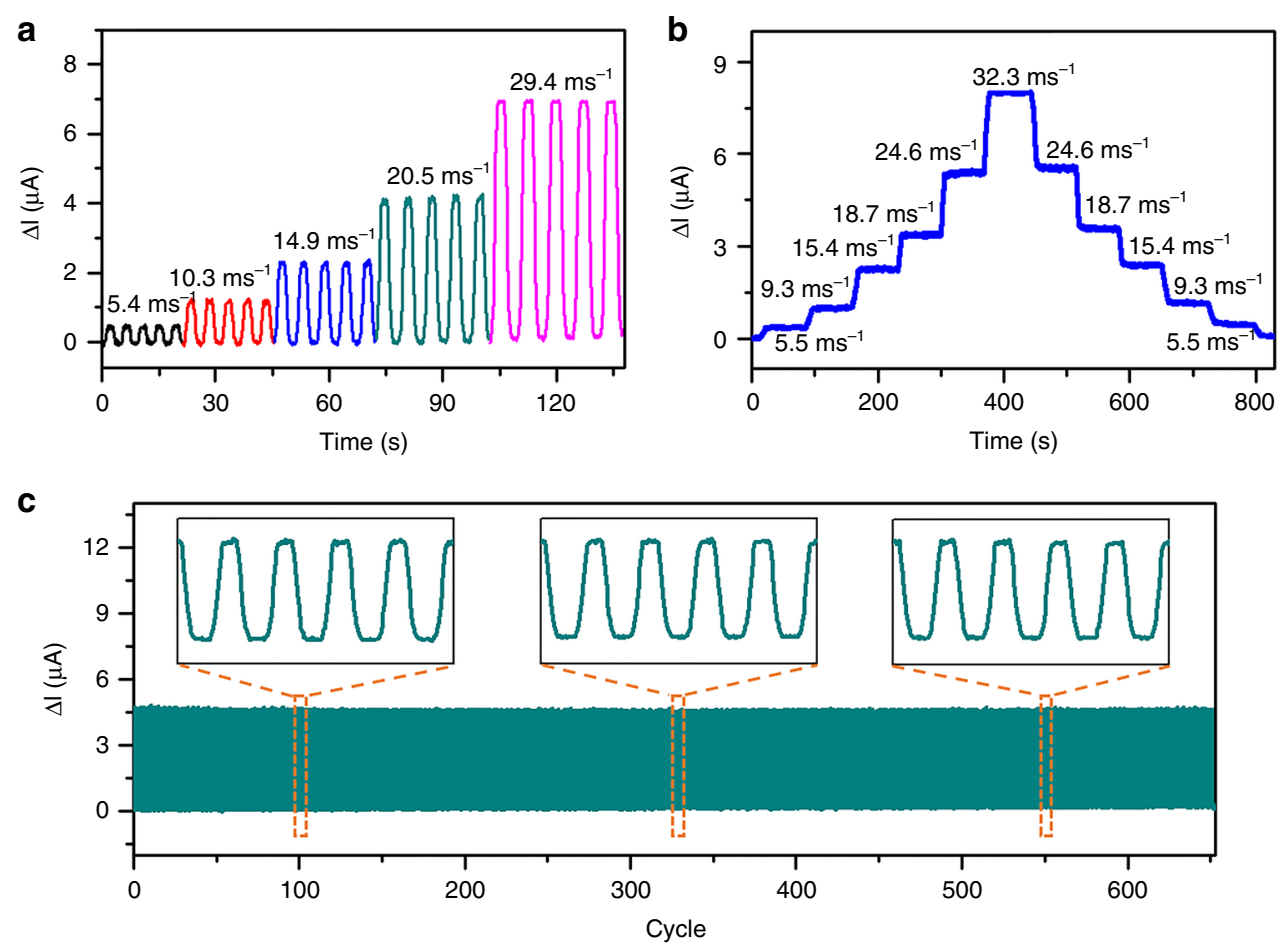

Fig. 4 Dynamics responses of the sensor. Measured photocurrent response of the sensor measured under $\mathbf{a}$ continuous and $\mathbf{b}$ instantaneous airflow changes. c Reliability measurement of the sensor at 650 cycles at a frequency of $0.2 \mathrm{~Hz}$

suggesting that the sensor is less sensitive with increasing rotation angle.

The dynamic responses of the sensor are studied by acquiring the photocurrent variation with time under different airflow conditions. Figure 4a shows the photocurrent response when the airflow rate is repeated in the range of 5.4-29.4 $\mathrm{ms}^{-1}$, indicating that the sensor exhibits a high degree of repeatability. The sensor response is then investigated by continuously increasing and decreasing the airflow between 5.5 and $32.3 \mathrm{~ms}^{-1}$, and the stepwise profiles of the photocurrent are observed in Fig. 4b. In addition, the reproducibility and stability of the sensor are evaluated by cyclic tests at a frequency of $0.2 \mathrm{~Hz}$. As shown in Fig. 4c, the output photocurrent signals are highly stable over 650 cycles, and the photocurrent waveforms in different periods are consistent.

To analyze the temporal response of the sensor, the PD is connected to a current amplifier (SR570, Stanford Research Systems) and an oscilloscope (MDO32, Tektronix), and the LED current remains fixed at $5 \mathrm{~mA}$. Figure $5 \mathrm{a}$ displays the transient response in the photocurrent when the airflow rate of $43.5 \mathrm{~ms}^{-1}$ is turned on and off. From the enlarged plot shown in Fig. 5b, the response time T90 and recovery time T10, defined as the time taken to reach 90 and $10 \%$ of the steady levels, are determined to be $12 \mathrm{~ms}$. Highly similar transient responses are also observed at other airflow rates (see Supplementary information).
Figure $5 \mathrm{c}$ summarizes the response time and measurement range of the reported airflow sensors. Compared with the sensors with response times below $1 \mathrm{~s}$, the sensor in this work provides a wider measurement range up to $53.5 \mathrm{~ms}^{-1}$. It is worth noting that the measured response time $(12 \mathrm{~ms})$ is more than three times shorter than the shortest value $(40 \mathrm{~ms})$ previously reported in the literature ${ }^{6}$. This remarkable performance can be attributed to the fast photoelectric conversion in the InGaN/ GaN MQW diode structure, and the transient response to the electrical pulse signal is as low as $1.2 \mu \mathrm{s}$ (see Supplementary Information), which is $2-3$ orders of magnitude smaller than that of the previously reported sensor (at submillisecond scales) ${ }^{11,13}$.

The proposed airflow sensor provides the attractive features of compact size, fast response, and wide detectable range and has a high potential for in situ measurement applications. For instance, airflow exhaled from the nose and mouth is considered a useful parameter for assessing human health ${ }^{38,39}$. To demonstrate its applicability in this area, the sensor is placed close to a person's nose, with a separation of $\sim 5 \mathrm{~cm}$, as shown in Fig. 6a. From the plot in Fig. $6 \mathrm{~b}$, the periodic change in photocurrent related to the respiratory rate is observed, and the signal varying in the range of $0.4 \mu \mathrm{A}$ implies that the airflow generated by nasal exhalation corresponds to a low airflow level. Under fast and deep breathing, a dense periodic photocurrent signal 

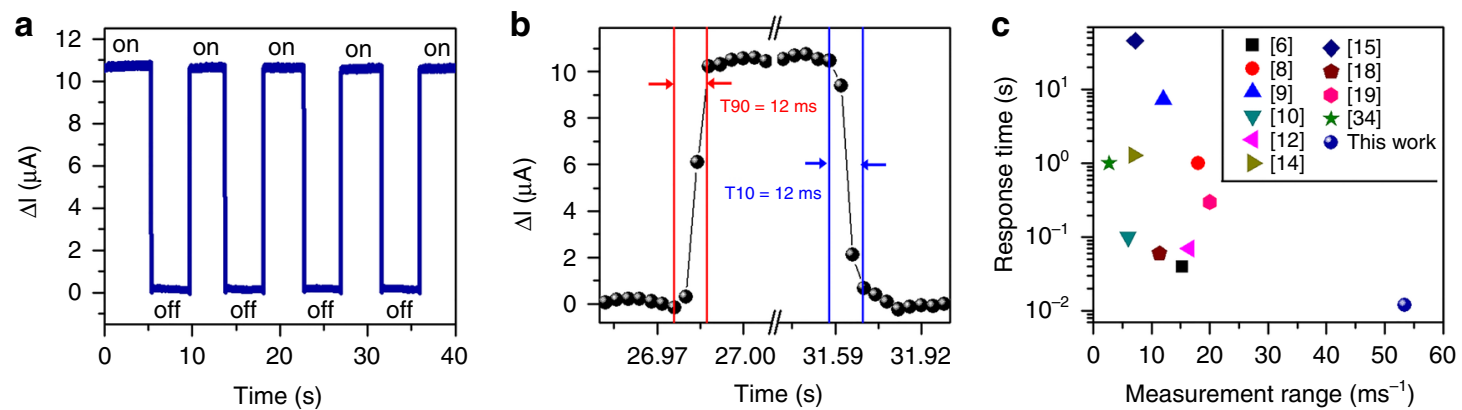

Fig. 5 Response time of the sensor. a Temporal responses of the sensor. $\mathbf{b}$ Enlarged view of the temporal response showing the response time and recovery time. c Comparison of the response time and the measurement range with those of other reported airflow sensors
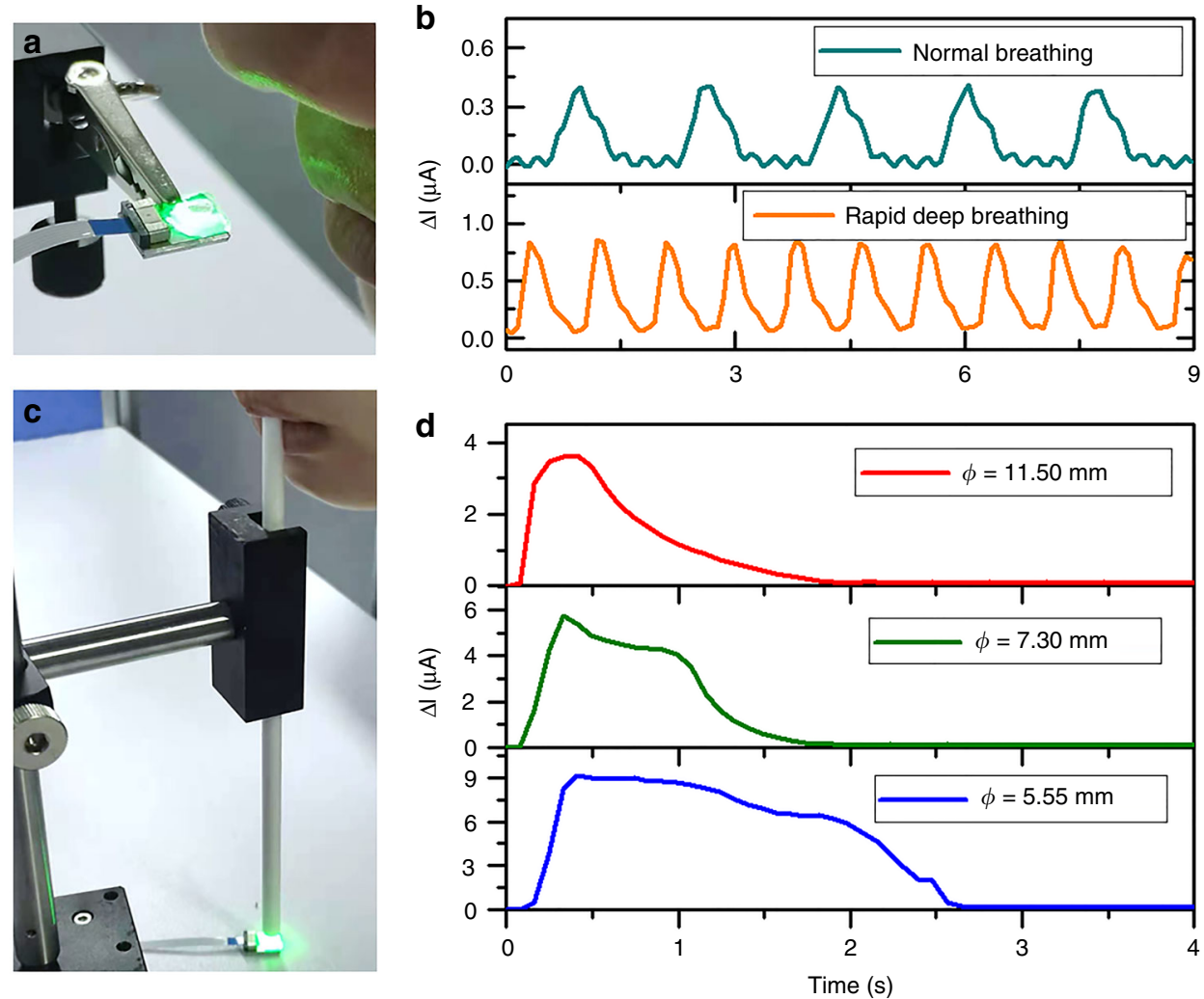

Fig. 6 Measurement of human exhaled airflow. Optical images show a person exhaling through the $\mathbf{a}$ nose and $\mathbf{c}$ mouth with the aid of a tube to the sensor. The photocurrent response of $\mathbf{b}$ nose breathing and $\mathbf{d}$ mouth blowing to the sensor through tubes with varying diameters ( $\varnothing)$ of 11.50 , 7.30, and $5.55 \mathrm{~mm}$

with a change of up to $8 \mu \mathrm{A}$ can be obtained. In addition to normal breathing patterns, determining the peak flow rate of a person's exhalation indicates how air is being expelled from the lungs, thereby judging the condition of asthma $^{40,41}$. Figure $6 \mathrm{c}$ shows the airflow measurement of a person exhaling air through a tube. The photocurrents from the sensor incorporating tubes with $\varnothing=11.50,7.30$, and $5.55 \mathrm{~mm}$ are plotted in Fig. 6d. Obviously, the photocurrent rises rapidly to the maximum value and decreases over time, representing a single continuous exhalation. It is found that a smaller $ø$ results in a broader decay curve. The tube with the smallest $\varnothing$ of $5.55 \mathrm{~mm}$ can concentrate the airflow to the sensor, leading to the maximum photocurrent change of $\sim 9 \mu \mathrm{A}$.

\section{Conclusion}

In summary, we present the development of an optical airflow sensor based on the integration of a GaN chip 
with a PDMS membrane in a controllable and scalable manner. Flexible PDMS highly responsive to airflow can modulate the light emitted from the chip without the aid of external optics, and the detected photocurrent signals can reflect changes in airflow. The proposed airflow sensor exhibits outstanding performance in terms of response time and detectable range, making it suitable for in situ measurement in a wide range of practical applications.

\section{Materials and methods \\ Fabrication of GaN chip}

Six-micrometer-thick epitaxial structures consisting of 9 pairs of $\operatorname{InGaN}(2.5 \mathrm{~nm}) / \mathrm{GaN}(12.5 \mathrm{~nm}) \mathrm{MQW}$ are grown by metal-organic chemical vapor deposition on a 4-in. c-plane sapphire substrate. A square mesa of $1000 \times$ $1000 \mu \mathrm{m}^{2}$ and a hexagonal mesa with a side length of $155 \mu \mathrm{m}$ are defined as a photodetector (PD) and lightemitting diode (LED), respectively, by photolithography. The unmasked GaN areas are etched to expose n-GaN using an inductively coupled plasma (ICP). To establish electrical isolation between the on-chip devices, a $10-\mu \mathrm{m}$ wide $\mathrm{GaN}$ region between the LED and PD is entirely removed by photolithography and ICP etching, followed by the deposition of a $\mathrm{SiO}_{2}$ passivation layer. The DBR acting as a bottom mirror is deposited by an optical thin-film coater. The p-pad and n-pad are deposited by electron-beam evaporation. After lapping and polishing of the sapphire face of the wafer, the chip with a size of $1 \times$ $1 \times 0.2 \mathrm{~mm}^{3}$ is diced by laser micromachining and then bonded on an aluminum-based PCB package. A detailed description of the process flow is provided in the Supplementary Information.

\section{Preparation of the PDMS membrane}

The process begins with the use of a micropipette to dispense drops of $40 \mu \mathrm{L}$ of deionized water into a 1-in. plastic petri dish. Because of the inherent hydrophobicity of the dish, the water droplets form a discrete hemispherical shape. The PDMS gel (Dow Sylgard 184) is prepared by mixing elastomer base and curing agent at a ratio of 10:1, followed by a degassing process in a vacuum chamber. The 1.35-g PDMS gel is poured over the droplets and allowed to rest for $10 \mathrm{~min}$. As the PDMS gel with lower density is immiscible with water, the coated PDMS self-flattens over time and forms a thin membrane geometry above the water drops. The sample is then loaded in an oven at $80^{\circ} \mathrm{C}$ for 2 hours for curing. The diameter and height of the PDMS circular cavity structure are 4.02 and $0.39 \mathrm{~mm}$, respectively, and the membrane thickness is $0.01 \mathrm{~mm}$. An $\mathrm{Al}$ reflective film with a diameter of $2 \mathrm{~mm}$ and a thickness of $0.01 \mathrm{~mm}$ is then formed by shaping the aluminum foil using a hole puncher.

\section{Acknowledgements}

The authors acknowledge financial support from the National Natural Science Foundation of China (62004088 and 12074170), the Shenzhen Natural Science Foundation Stability Support Program Project (20200925160044004), and NSQKJJ (K21799120)

\section{Author details}

${ }^{1}$ School of Microelectronics, Southern University of Science and Technology, Shenzhen 518055, China. ${ }^{2}$ Engineering Research Center of Integrated Circuits for Next-Generation Communications, Ministry of Education, Southern University of Science and Technology, Shenzhen 518055, China. ${ }^{3}$ Engineering Research Center of Three Dimensional Integration in Guangdong Province, Southern University of Science and Technology, Shenzhen 518055, China

\section{Author contributions}

Y.L., X.A., and K.H.L. conceived the idea of the paper. Y.L., X.A., and L.C. performed the experiments and carried out the data analysis. K.H.L. wrote the paper, and Y.L. assisted. L.C. and K.H.L. guided and supervised the work. All authors contributed to the general discussion and approved the final paper.

\section{Data availability}

The data supporting plots within this paper and other findings of this study are available from the corresponding author upon request.

\section{Conflict of interest}

The authors declare no competing interests.

Supplementary information The online version contains supplementary material available at https://doi.org/10.1038/s41378-021-00335-1.

Received: 1 September 2021 Revised: 25 October 2021 Accepted: 9 November 2021

Published online: 04 January 2022

\section{References}

1. Zhu, Y. X., Luo, M. H., Ouyang, Q., Huang, L. \& Cao, B. Dynamic characteristics and comfort assessment of airflows in indoor environments: a review. Build Environ. 91, 5-14 (2015).

2. Eccles, R. Nasal airflow in health and disease. Acta Oto-Laryngol. 120, 580-595 (2000).

3. Walker, I. J. \& Nickling, W. G. Dynamics of secondary airflow and sediment transport over and in the lee of transverse dunes. Prog. Phys. Geog. 26, 47-75 (2002).

4. Chu, W. X. \& Wang, C. C. A review on airflow management in data centers. Appl. Energy 240, 84-119 (2019).

5. Smale, N. J., Moureh, J. \& Cortella, G. A review of numerical models of airflow in refrigerated food applications. Int. J. Refrig. 29, 911-930 (2006).

6. Huang, S. Y., Zhang, B. C., Lin, Y., Lee, C. S. \& Zhang, X. H. Compact biomimetic hair sensors based on single silicon nanowires for ultrafast and highly-sensitive airflow detection. Nano Lett. 21, 4684-4691 (2021).

7. Park, J. et al. Giant tunneling piezoresistance of composite elastomers with interlocked microdome arrays for ultrasensitive and multimodal electronic skins. ACS Nano 8, 4689-4697 (2014).

8. Takahashi, H., Nakai, A. \& Shimoyama, I. Waterproof airflow sensor for seabird bio-logging using a highly sensitive differential pressure sensor and nano-hole array. Sens. Actuators A 281, 243-249 (2018).

9. Bian, Y. X., Liu, R. R. \& Hui, S. Fabrication of a polyvinylidene difluoride fiber with a metal core and its application as directional air flow sensor. Funct. Mater. Lett. 9, 1650001 (2016).

10. Zhao, S. \& Zhu, R. Electronic skin with multifunction sensors based on thermosensation. Adv. Mater. 29, 1606151 (2017).

11. Talbi, A. et al. A micro-scale hot wire anemometer based on low stress (Ni/W) multi-layers deposited on nano-crystalline diamond for air flow sensing. J. Micromech. Microeng. 25, 125029 (2015).

12. Sadeghi, M. M., Peterson, R. L. \& Najafi, K. Air flow sensing using micro-wirebonded hair-like hot-wire anemometry. J. Micromech. Microeng. 23, 085017 (2013).

13. Neda, T., Nakamura, K. \& Takumi, T. A polysilicon flow sensor for gas flow meters. Sens. Actuators A 54, 626-631 (1996). 
14. Wang, H. M. et al. Bioinspired fluffy fabric with in situ grown carbon nanotubes for ultrasensitive wearable airflow sensor. Adv. Mater. 32, 1901980 (2020).

15. Xu, Z. Y. et al. Highly sensitive airflow sensors with an ultrathin reduced graphene oxide film inspired by gas exfoliation of graphite oxide. Mater. Horiz. 4, 383-388 (2017).

16. Liu, Y. F. et al. A biomimetic multifunctional electronic hair sensor. J. Mater. Chem. A 7, 1889-1896 (2019).

17. Zhao, S. F. et al. 3D dielectric layer enabled highly sensitive capacitive pressure sensors for wearable electronics. ACS Appl. Mater. Interfaces 12, 32023-32030 (2020).

18. Wu, Z. H. et al. Flexible out-of-plane wind sensors with a self-powered feature inspired by fine hairs of the spider. ACS Appl. Mater. Interfaces 11, 44865-44873 (2019).

19. Su, L. et al. Low detection limit and high sensitivity wind speed sensor based on triboelectrification-induced electroluminescence. Adv. Sci. 6, 1901980 (2019).

20. Yin, M. J. et al. Recent development of fiber-optic chemical sensors and biosensors: mechanisms, materials, micro/nano-fabrications and applications. Coord. Chem. Rev. 376, 348-392 (2018).

21. Ramakrishnan, M., Rajan, G., Semenova, Y. \& Farrell, G. Overview of fiber optic sensor technologies for strain/temperature sensing applications in composite materials. Sensors 16, 99 (2016).

22. Wang, X. H. et al. Optical fiber anemometer using silver-coated fiber Bragg grating and bitaper. Sens. Actuators A 214, 230-233 (2014).

23. Wang, P., Zhao, Y., Yang, Y. \& Bi, D. J. Research on a novel variable-area optical fiber gas flow sensor. Sens. Actuators A 221, 29-32 (2015).

24. Zhao, Y., Wang, P., LV, R. Q. \& Liu, X. Highly sensitive airflow sensor based on Fabry-Perot interferometer and Vernier effect. J. Lightwave Technol. 34, 5351-5356 (2016).

25. Lee, C. L., Yang, C. X., Liang, T. Y. \& Ma, C. T. Novel airflow sensor using laser heated Sn-microsphere airgap fiber Fabry-Perot interferometer. IEEE Photonic Tech. Lett. 31, 1775-1778 (2019).

26. Cheri, M. S. et al. Fabrication, characterization, and simulation of a cantileverbased airflow sensor integrated with optical fiber. Appl. Opt. 52, 3420-3427 (2013).
27. Kang, $Y$. et al. Nanostructured optical fibre sensors for breathing airflow monitoring. Meas. Sci. Technol. 17, 1207-1210 (2006).

28. Akasaki, I. \& Amano, H. Breakthroughs in improving crystal quality of GaN and invention of the p-n junction blue-light-emitting diode. Jpn J. Appl Phys. 45 9001-9010 (2006).

29. Wong, M. S., Nakamura, S., DenBaars, S. P. Review-progress in high performance III-nitride micro-light-emitting diodes. ECS J. Solid State Sci. 9, 015012 (2019).

30. Wang, Y. J. et al. Full-duplex light communication with a monolithic multicomponent system. Light Sci. Appl. 7, 83 (2018).

31. Li, K. H. et al. Monolithically integrated InGaN/GaN light-emitting diodes, photodetectors, and waveguides on Si substrate. Optica 5, 564-569 (2018).

32. Chen, L., Wu, Y. P. \& Li, K. H. Monolithic InGaN/GaN photonic chips for heart pulse monitoring. Opt. Lett. 45, 4992-4995 (2020).

33. $\mathrm{Fu}, \mathrm{K}$. et al. Simultaneous illumination-imaging. Adv. Mater. Technol. 2100227 (2021).

34. Zhang, S. et al. Membrane light-emitting diode flow sensor. Adv. Mater. Technol. 3, 1700285 (2018).

35. Qin, C. et al. Transferrable monolithic multicomponent system for nearultraviolet optoelectronics. Appl. Phys. Express 11, 051201 (2018).

36. Cheung, Y. F., Li, K. H. \& Choi, H. W. Flexible free-standing III-nitride thin films for emitters and displays. ACS Appl. Mater. Interfaces 8, 21440-21445 (2016).

37. Li, K. H., Lu, H. T., Fu, W. Y., Cheun, Y. F. \& Choi, H. W. Intensity-stabilized LEDs with monolithically integrated photodetectors. IEEE Trans. Ind. Electron. 66, 7426-7432 (2019).

38. Gupta, J. K., Lin, C. H. \& Chen, Q. Y. Characterizing exhaled airflow from breathing and talking. Indoor Air 20, 31-39 (2010).

39. Holmer, I., Kuklane, K. \& Gao, C. S. Minute volumes and inspiratory flow rates during exhaustive treadmill walking using respirators. Ann. Occup. Hyg. 51, 327-335 (2007).

40. Benchetrit, G. Breathing pattern in humans: diversity and individuality. Resp Physiol. 122, 123-129 (2000).

41. Neerincx, A. H. et al. Breathomics from exhaled volatile organic compounds in pediatric asthma. Pediatr. Pulm. 52, 1616-1627 (2017). 